\title{
Über den Insulinabbau in der isolierten Rattenleber bei Perfusion mit Rattenserum und Leucin*
}

\author{
K. Kopetz, H. BÜrgi, E. R. Froensch und K. Schwarz
}

II. Medizinische Klinik der Universität München (Direktor: Prof. Dr. Dr. G. BodechtwL) und Stoffwechselabteilung (Leiter: Prof. Dr. A. LABHART) der Medizinischen Klinik der Universität Zürich (Direktor: Prof. Dr. P.H. Rossier)

Eingegangen am 16. Februar 1966

The degradation of insulin by the isolated rat liver perfused with rat serum and leucine.

Summary. 1. Isolated rat livers were perfused with a semisynthetic medium containing diluted rat serum to see whether or not the rat liver can convert crystalline rat insulin into nonsuppressible insulin-like activity. The influence of L-loucine on the clearance of insulin by the perfused liver was studied in the same perfusion system. - 2. Nonsuppressible ILA was not found in the perfusion fluid, even after prolonged perfusion of the liver with crystalline rat insulin. -3 . The clearance of insulin by the liver was not changed by the addition of L-leucine (approximately $60 \mathrm{mg} \%$ ) to the perfusion fluid. - 4. The specific liver clearances for crystalline insulin were almost identical under the 3 experimental conditions studied. The perfused isolated rat liver appears to be a good model for the study of the hepatic degradation of insulin.

Dégradation de l'insuline dans le foie isolé de rat lors de la perfusion avec du sérum de rat et de la leucine.

Résumé. Des foies isolés de rats ont été perfusés avec un milieu semisynthétique contenant du sérum dilué de rat, pour voir si le foie de rat peut ou non convertir l'insuline cristallisée de rat en activité insulinique non supprimable. - L'influence de la L-Ieucine sur la clearance de l'insuline par le foie perfusé a été étudiée avec le même système de perfusion. - 2. On n'a pas trouvé de I.L.A. non supprimable dans le liquide de perfusion, même après perfusion prolongée du foie avec l'insuline cristallisée de rat. - 3. La clearance de l'insuline par le foie n'était pas modifiée par l'addition de L-leucine (approximativement $60 \mathrm{mg} \mathrm{\%}$ ) au liquide de perfusion. - 4. Les clearances spécifiques du foie pour l'insuline cristallisée étaient pratiquement identiques dans les 3 conditions expérimentales étudiées. Le foie de rat isolé perfusé paraît être une bonne préparation pour l'étude de la dégradation hépatique de l'insuline.

Zusammenfassung. 1. Mit Hilfe der zyclischen Perfusion isolierter Rattenlebern mit verdünntem Rattenserum wurde versucht, zur Frage der Umwandlung von kristallinem Ratteninsulin in die nicht-antikörperhemmbare Insulinaktivität (NHI) in der Leber Stellung zu nehmen. In weiteren Versuchen wurde der Einfluß von Leucin auf den Insulinabbau in der Leber geprüft. - 2. Wie schon bei der Verwendung eines halbsynthetischen Perfusionsmediums konnte nach Durchströmung mit verdünntem Rattenserum keine Umwandlung von kristallinem Ratteninsulin in die NHI durch die isolierte Rattenleber festgestellt werden. - 3. Die Durchströmungsversuche ergaben keinen Hinweis auf eine Veränderung des Insulinabbaus in der Leber unter dem Einfluß von L-Leucin. 4. Die aufgrund der Durchströmung isolierter Rattenlebern ermittelte Leberclearance und spezifische Leberclearance des Insulins zeigte unter verschiedenen Versuchsbedingungen eine gute Übereinstimmung. Die Methode der Perfusion der isolierten Rattenleber ist deshalb als geeignetes Modell zur Untersuchung des Insulinabbaus anzusehen.
Untersuchungen der letzten Jahre haben unter Verwendung biologischer Methoden Aufschlüsse über die Natur der im Serum vorhandenen Insulinaktivität erbracht. Diese läßt sich durch Messung am isolierten Fettgewebe aufteilen in eine durch Antikörper hemmbare (HI) und in eine durch Antikörper nicht hemmbare Form (NHI). Aufgrund der Befunde von BÜBGr et al. [4] und Froesch [12] et al. beim Inselzelladenom und der Untersuchungen von SAMAAN et al. [30, 31] ist anzunehmen, daß Insulin von den B-Zellen der Pankreasinseln in hemmbarer Form sezerniert wird. Die HI ist mit dem immunologisch nachweisbaren Insulin identisch [36]. Die NHI ist in der Elektrophorese den Alpha $a_{2}-\beta_{1}$-Globulinen assoziiert.

Die Bildung der NHI wurde von SamaAN et al. in die Leber verlegt. Diese Autoren fanden bei Patienten mit Leberinsuffizienz erniedrigte Werte der NHI und

* Mit Unterstützung der Deutschen Forschungsgemeinschaft (Ko 225) und des Sehweizerischen Nationalfonds zur Förderung der Wissenschaften. nach Durchströmung der Leber pankreatektomierter Hunde mit Altinsulin einen Anstieg der NHI im Venenblut [32, 33]. In früheren Untersuchungen haben wir mit der Methode der Perfusion isolierter Rattenlebern keinen Anhalt für eine Umwandlung der HI in NHI in der Leber finden können [5]. Diese Perfusionen wurden jedoch mit einem halbsynthetischen Medium bestehend aus Rinderalbumin, Tyrodelösung und Glucose durchgeführt. Die Vergleichbarkeit des Modells mit physiologischen Verhältnissen war somit neben der Isolierung des Organs durch die Art des Perfusionsmediums eingeschränkt. Zum Ausschluß der Möglichkeit, daß bei der Umwandlung der HI in NHI in der Leber Plasmaproteine eine maßgebende Rolle spielen oder in dem isolierten Organ eine Bindung an Plasmaproteine erfolgt, haben wir in der vorliegenden Arbeit die gleiche Frage mithilfe der Perfusion isolierter Rattenlebern mit verdünntem Rattenserum untersucht. In einer zweiten Gruppe von Perfusionen versuchten wir, eine Antwort auf die Frage nach dem Ein- 
fluß von Leucin auf den Insulinabbau bzw. die Umwandlung von HI in NHI in der Leber zu erhalten.

Leucin führt bei empfindlichen Säuglingen und Kleinkindern, bei einem Teil der Patienten mit Inselzelladenomen und, wesentlich schwächer aber dennoch signifikant, auch bei gesunden Versuchspersonen zu einer Senkung des Blutzuckerspiegels.

\section{Methoden}

Die zur Durchströmung verwendeten Organe wurden männlichen Ratten eines Sprague-Dawleystammes mit einem Körpergewicht von $240-270 \mathrm{~g}$ in leichter Nembutalnarkose entnommen. Die Perfusion wurde nach der Methode von MrLLeR [22] in einer Modifikation von ScHIMLASSEK [34] durchgeführt. Als Kontrollen des invivo-status der Leber diente die laufende Untersuchung des Lactat-Pyruvatquotienten im Perfusionsmedium und des ATP/ADP-Quotienten im Lebergewebe nach Abschluß der Perfusion. Versuche, die den von SchimasseK geforderten Kriterien nicht genügten, wurden bei der Auswertung nicht berücksichtigt.

Das Perfusionsmedium bestand bei Gruppe A, über die wir an anderer Stelle bereits berichteten [5], aus Rindererythrozyten (Haematokrit 31-34\%), Rinderalbu$\min (2 \mathrm{~g} / 100 \mathrm{ml})$, Tyrodelösung und Glucose $(100 \mathrm{mg} \%)$ mit einem $\mathbf{p H}$ von 7.4. Die Organe der Versuchsgruppe B wurden mit Rattenserum, verdünnt mit Tyrodelösung 1:2 perfundiert. Das Rattenserum stammte von OsborneMendel-Ratten, die nach einer Fastenzeit von 48 Stunden in $\mathrm{CO}_{2}$-Narkose durch Aortenpunktion entblutet wurden. Dieses Serum enthielt weniger als $50 \mu \mathrm{E}$ Insulinaktivität pro $\mathrm{ml}$.

Das Perfusionsmedium in Gruppe $\mathrm{C}$ war identisch mit dem halbsynthetischen Medium der Gruppe A, enthielt aber zusätzlich L-Leucin. ${ }^{1}$ Leucin wurde dem Perfusionsmedium in einer Menge von $50 \mathrm{mg} \%$ zugesetzt und anschließend wurden während des ganzen Versuches $6.92 \mathrm{mg}$ Leucin gelöst in Tyrodelösung pro Stunde durch eine elektrische Pumpe kontinuierlich in das System infundiert um einen möglichst konstanten Spiegel zu erhalten. Die Leucinkonzentration im erythrozytenfreien Perfusat wurde bei einzelnen Perfusionen durch Bestimmung mit dem Stein and Moore Aminoacidanalyzer kontrolliert. ${ }^{2}$ Ratteninsulin wurde in 1/250 $\mathrm{n} \mathrm{HCl}$ gelöst und dem Perfusat zugesetzt, bei Gruppe A 0.2 resp. $2 \mathrm{E}$, Gruppe B $2.0 \mathrm{E}$ resp. $0.75 \mathrm{E}$ und bei Gruppe $\mathrm{C} 2.0 \mathrm{E}$.

Die einzelnen Versuche dauerten 4 (Gruppe B, C) bzw. 6 (Gruppe A) Stunden. Das Anfangsvolumen der Perfusionslösung betrug $200 \mathrm{ml}$ und wurde bei den verschiedenen Abnahmen $(30,60,90,120,150,180,210$ und 240 Minuten) um jeweils $8 \mathrm{ml}$ vermindert.

Die Bestimmung der Insulinaktivitäten im erythrozytenfreien Perfusat erfolgte nach der Methode von Froesch et al. [12] in der von Bürar et al. [5] angegebenen Weise. Die kleinste Verdünnung des Perfusats betrug $1: 3$; die geringste meßbare ILA-Konzentration ${ }^{3}$ war demnach $30 \mu \mathrm{E} / \mathrm{ml}$.

Die Leberclearance $K$ wurde nach folgender allgemeiner Formel errechnet:

$$
K=\frac{2.3 V_{\text {perf }}}{\mathrm{t}} \times \log \cdot \frac{C_{a}}{C_{f}}
$$

1 Für die Überlassung von L-Leucin sind wir der Firma Deutsche Hoffmann La Roche zu Dank verpflichtet.

${ }_{2}^{2}$ Für die Durchführung der Bestimmung sind wir Herrn Dr. R.E. Humber vom Biochemischen Institut der Universität Zürich zu Dank verpflichtet.

3 Unter ILA ist die am Fettgewebe gemessene Insulinaktivität $\mathrm{zu}$ verstehen.
Wobei:

$K=$ Leberclearance $(\mathrm{ml} / \mathrm{min})$

$V_{\text {perp }}=$ Volumen der Perfusionsflüssigkeit während der Periode $t_{\alpha}$ bis $t_{f}(\mathrm{ml})$

$t \quad=$ Zeit (min)

$\Delta t=t_{f}-t_{a}(\min )$

$C_{a}=$ Insulinkonzentration zur Zeit $t_{a}(\mu \mathrm{E} / \mathrm{ml})$

$C_{f}=$ Insulinkonzentration zur Zeit $t_{f}(\mu \mathrm{E} / \mathrm{ml})$

Bei den Perfusionen mit Leucin komplizierte sich die Berechnung, weil durch die kontinuierliche Infusion von leucinhaltiger aber insulinfreior Perfusionslösung (rund $4 \mathrm{ml} / \mathrm{Std}$ ) das Insulin im Perfusat laufond verdünnt wurde. Dieser Verdünnung muß bei der Berechnung der Leberelearance mit folgender approximativer Formel Rechnung getragen werden:

$C_{\text {corr }}=$ Insulinkonzentration, die an dem bestimmten Zeitpunkt erreicht worden wäre, wenn keine Verdünnung durch die kontinuierliche Infusion stattgefunden hätte.

Dementsprechend änderte sich die Formel:

$$
K=\frac{2.3 V_{\text {perf }}}{t} \times \log \cdot \frac{C_{a}}{C_{f \text { corr }}}
$$

Da bei den verschiedenen Perfusionstypen die Lebergewichte nicht identisch waren, mußte die spezifische Leberclearance errechnet werden:

$$
\text { Spezif. Leberclearance }=K_{\text {spec }}=\frac{K}{\text { Lebergewicht }}
$$

Tabelle 1. Leberperfusion mit L-Leucinhaltigem Medium (Gruppe C). Daten für die Berechnung der Leberclearance. Jeder Wert für die totale Insulinaktivität entspricht einem Mittelwert aus drei Versuchen. SEM =, standard error of the mean" = Standardabweichung des Mittelwertes

Zeit der Entnahme (Minuten nach Zugabe des Insulins)

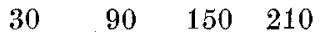

Totale Insulinaktivität im Perfusat $(\mu \mathrm{E} / \mathrm{ml})$

$6700 \quad 3280 \quad 1790 \quad 630$

Korrigierte totale Insulinaktivität im Perfusat $(\mu \mathrm{E} / \mathrm{mI})$

Mittlere Perfusionsvolumen im Intervall zwischen den Entnahmen (ml)

Im Intervall zwischen den Entnahmen infundierte Perfusionsflüssigkeit (ml)

Leberclearance im Intervall zwischen den Entnahmen (ml/min)

$\begin{array}{llll}2.14 & 1.67 & 2.73 & 2.18 \\ & & & 0.30\end{array}$

\section{Ergebnisse}

Tabelle 1 gibt die Werte der Konzentration der totalen Insulinaktivität und der Leberclearances in den Perfusionen der Gruppe $\mathrm{C}$ wieder. Die mittlere Leberclearance beträgt in diesen Versuchen $2.18 \mathrm{ml} / \mathrm{min}$. Bei einem mitt. leren Lebergewicht von $11 \mathrm{~g}$ ergibt sich eine spezifische Leberclearance von $0.20 \pm 0.03 \mathrm{ml} / \mathrm{min} / \mathrm{g}$ (Mittelwert \pm Standardabweichung des Mittelwertes). Die an anderer Stelle [5] veröffentlichten Werte der Gruppe A hatten eine spezifische Leberclearance von $0.18 \mathrm{ml} / \mathrm{min} / \mathrm{g}$ ergeben. Dieser Wert ist vom oben angegebenen Wert der Gruppe $\mathrm{C}$ nicht signifikant verschieden. In Abbildung 1 ist der Insulinabbau im leucinhaltigen Perfusat graphisch dargestellt. In einem semilogarithmischen System liegen die Punkte, wie bei der Gruppe A [5], annähernd auf einer geraden Linie. Aus dem unteren Teil der Abbildung 1 ist 
ersichtlich, daß dank kontinuierlicher Leucinzufuhr während der ganzen Perfusionsdauer eine konstante Leucinkonzentration beibehalten wird.

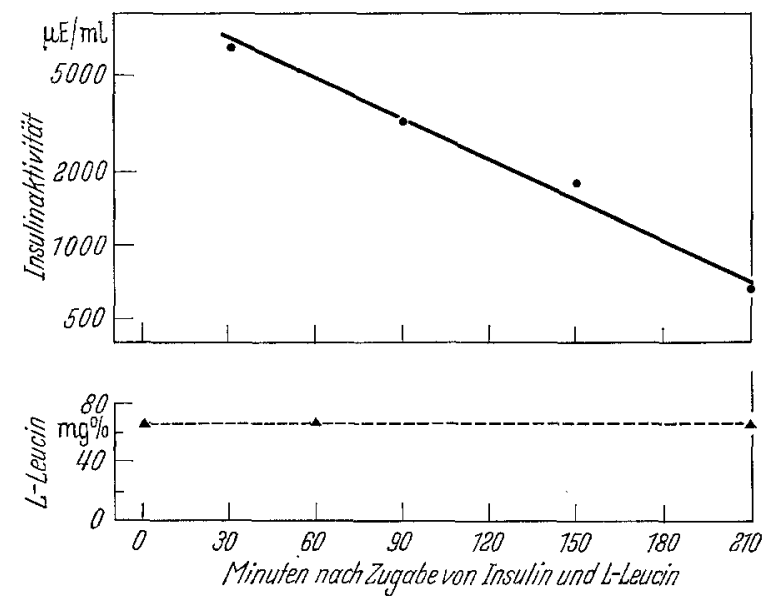

Abb. 1. Totale Insulinaktivität und Leucinkonzentration während Leberperfusionen mit leucinhaltigem Medium (Gruppe C). Zur Zeit 0 wurden $2.0 \mathrm{E}$ Ratteninsulin zu $200 \mathrm{ml}$ Perfusionsflüssigleit zugesetzt. Jeder Punkt für die Insulinaktivität stellt einen Mittelwert aus drei Versuchen dar. Die ausgezogene Linie entspricht der Regressionsgeraden der vier Punkte. Die gestrichelte Linie im unteren Teil der Abbildung gibt die Leucinkonzentration im Perfusat wieder, wobei jeder Punkt dem Mittelwert aus zwei Versuchen entspricht

Tabelle 2. Perfusion der Leber mit Rattenserum enthaltendem Medium (Gruppe B). Daten für die Berechnung der Leberclearance. Jeder Wert für die totale Insulinaktivität entspricht einem Mittelwert aus drei Versuchen. $S E M=$ "standard error of the mean" $=$ Standardabweichung des Mittelwertes

Zeit der Entnahme (Minuten

nach Zugabe des Insulins)

$\begin{array}{llll}30 & 90 & 150 & 210\end{array}$

Totale Insulinaktivität

$(\mu \mathrm{E} / \mathrm{ml})$

$5460 \quad 2270 \quad 1500 \quad 370$

Perfusionsvolumen im

Intervall zwischen den

Entnahmen (ml

$\begin{array}{llll}129 & 122 & 115 & \begin{array}{l}\text { Mittel } \\ + \text { SEM }\end{array}\end{array}$

Leberclearance im Intervall zwischen den Entnahmen $(\mathrm{ml} / \mathrm{min})$

$\begin{array}{llll}1.88 & 0.83 & 2.68 & 1.80 \pm\end{array}$

Tabelle 2 enthält die Werte der totalen Insulinaktivität und die Leberclearances in den Perfusaten der Gruppe B. Die mittlere Leberclearance beträgt in dieser Gruppe $1.80 \mathrm{ml} / \mathrm{min}$, was bei einem mittleren Lebergewicht von $11 \mathrm{~g}$ eine spezifische Leberclearance von $0.16 \pm 0.05$ $\mathrm{ml} / \mathrm{min} / \mathrm{g}$ ergibt (Mittelwert \pm Standardabweichung des Mittelwertes). Diese spezifische Leberclearance ist von den beiden vorgenannten nicht signifikant verschieden. Abbildung 2 zeigt, daß die Punkte der Insulinkonzentration in einem semilogarithmischen System wie bei den Gruppen $A$ und $C$ annähernd auf einer Geraden liegen. Im unteren Teil der Abbildung 2 sind die Konzentrationen von NHI in zwei Perfusionen dargestellt. Außer zwei Meßwerten liegen alle Werte unter $30 \mu \mathrm{E} / \mathrm{ml}$, d.h. unter der üblicherweise mit unserer Methode noch meßbaren Konzentration. Die beiden Werte über $30 \mu \mathrm{E} / \mathrm{ml}$ erwiesen sich bei statistischer Prüfung der betreffenden InsulinAssays ebenfalls als nicht signifikant von Null verschie- den. Es steht somit fest, daß auch in Anwesenheit von Rattenserum in der Leberperfusion keine Umwandlung von kristallinem Insulin in NHI erfolgt.

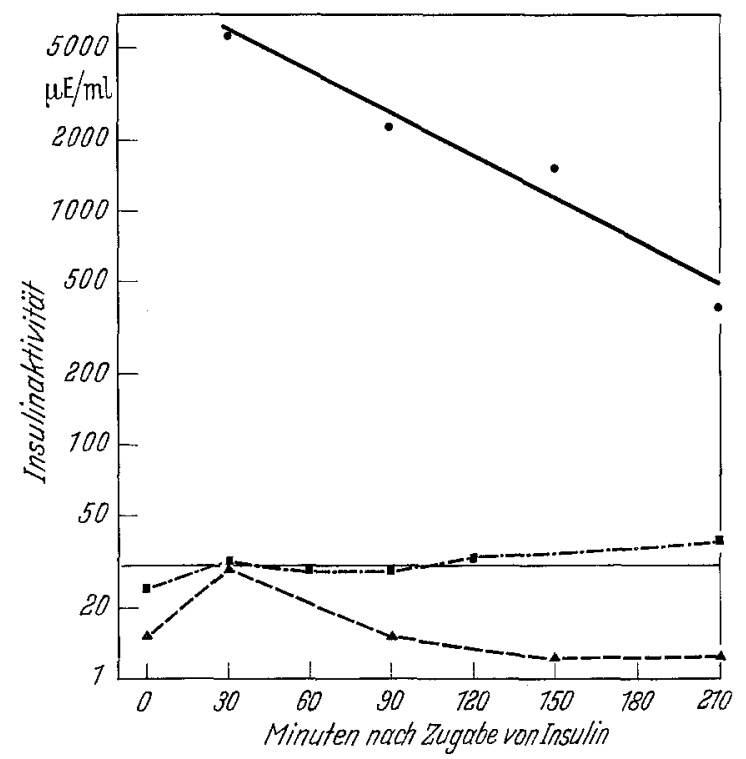

Abb. 2. Totale und nicht hemmbare Insulinaktivität während Leberperfusionen mit Medium, das Rattenserum enthält (Gruppe B). Jeder Punkt für die totale Insulinaktivität $(\bullet)$ entspricht dem Mittelwert aus drei Versuchen. Zur Zeit Null wurden 0.75 E Ratteninsulin zu $150 \mathrm{ml}$ Perfusionsfüssigkeit zugegeben. Die ausgezogene Linie stellt die Regressionsgerade für die totale Insulinaktivität dar. Die Punkte für die nicht hemmbare Insulinaktivität (G, A) stammen aus zwei Einzelversuchen, bei denen zur Zeit Null $2.0 \mathrm{E}$ Ratteninsulin zu $200 \mathrm{ml}$ Perfusionsfüssigkeit gegeben wurden. Die gestrichelte horizontale Linie entspricht der niedrigsten mit dem Assay noch meßbaren Instulinaktivität $(30 \mu \mathrm{E} / \mathrm{ml})$

Wir danken Fräulein UTE Westermayer, Fräulein Renate Zigch und Fräulein BrigtTte Schwarz für ihre unermüdliche und gewissenhafte technische Mithilfe bei dieser Arbeit.

\section{Diskussion}

Insulin wird vom Pankreas in einer Form, deren Aktivität durch Anti-Insulin-Serum hemmbar ist, abgegeben. Der größte Teil der Insulinaktivität des Serums ist indessen nicht hemmbar. Nach den bereits zitierten Befunden von SAMAaN et al. [32, 33], schien die Annahme berechtigt, daß die Leber Insulin in nicht hemmbare ILA umwandeln könnte. Da die Ratte einen ähnlichen Plasmaspiegel von HI und NHI wie der Mensch hat, prüften wir diese Hypothese mithilfe der Perfusion der isolierten Rattenleber. Frühere Experimente [5] hatten keine Umwandlung von kristallinem Insulin in NHI durch die mit einem halbsynthetischen, albuminhaltigen Medium perfundierten Rattenleber gezeigt. Um die Möglichkeit auszuschließen, daß diese Umwandlung von Insulin in NHI unter Mitwirkung von Plasmaproteinen abläuft, haben wir die gleichen Perfusionsversuche an isolierten Rattenlebern mit verdünntem Rattenserum wiederholt. Auch unter diesen Versuchsbedingungen war während der gesamten Versuchsdauer kein signifikanter Anstieg der NHI im Perfusionsmedium feststellbar. Diese Befunde 
passen zu den kürzlich publizierten Daten, wonach NHI mit größter Wahrscheinlichkeit kein Insulin und auch keine der beiden Insulinketten enthält [6]; sie stehen jedoch im Widerspruch zu den Beobachtungen von Sress et al. [35]. Diese Autoren wiesen bei Durchströmung der isolierten Rattenleber mit einem insulinfreien Medium einen laufenden Anstieg der ILA nach. Ob diese Diskrepanz auf Unterschieden im Perfusionsmedium, z.B. dem Zusatz von Zinksulfat, beruht, soll in weiteren Untersuchungen geklärt werden.

Unsere Untersuchungen ergaben Aufschlüsse über den Abbau der HI durch die perfundierte Leber. Über die Schwundrate des Plasmainsulins liegen eine Reihe von Untersuchungen, allerdings zum großen Teil mit $J^{131}$ markiertem Insulin, vor. Die Markierung mit diesem Isotop scheint nicht ohne Bedeutung für die Eigenschaften des Insulins zu sein, da nach Jodmarkierung eine Minderung der hypoglykaemischen Wirkung von Insulin gefunden wurde [4]. Weiterhin ist mit der Möglichkeit zu rechnen, daß nicht allein die Jodierung, sondern auch ionisierende Effekte der Gammastrahlung zu molekularen Veränderungen führt [2]. In diesem Zusammenhang ist die Beobachtung von Mortimore und Tietze [24] interessant, die bei cyclischer Perfusion der isolierten Rattenleber mit markiertem Insulin ein Sistieren des Insulinabbaus bei einem Spiegel von 30\% der Anfangskonzentration fanden. Demgegenüber zeigte sich in unseren Ver. suchen ein kontinuierlich fortschreitender Abbau der HI. Bei Verwendung einer semilogarithmischen Skala ergaben die nach jeweils einer Stunde Intervall ermittelten Konzentrationen einen annähernd geradlinigen Abfall. Sie lagen nach 3 Stunden Perfusion unter $10 \%$ der Ausgangskonzentration.

Die Leberclearance betrug in unseren Versuchen nach Perfusion mit einem halbsynthetischen Medium $2.67 \mathrm{ml} / \mathrm{min}$ und mit verdünntem Rattenserum $1.80 \mathrm{ml} /$ min. Der erste Wert entspricht annähernd dem von Mortimore et al. [25] gefundenen Mittelwert von $3.05 \mathrm{ml} / \mathrm{min}$. Die nach Durchströmung mit verdünntem Rattenserum beobachtete Leberclearance von $1.80 \mathrm{ml} /$ min war deutlich niedriger. Ein Vergleich der Werte ist jedoch nur begrenzt möglich, da in beiden Arbeiten unterschiedlich schwere Organe durchströmt wurden, das Lebergewicht aber nur bei der spezifischen Leberclearance berücksichtigt wird. Außerdem weichen die Versuchsanordnungen zum Teil erheblich voneinander ab. Montimore et al. verwendeten beispielsweise markiertes Insulin, Rattenvollblut und ein wesentlich kleineres Perfusionsvolumen $(50 \mathrm{ml})$.

Bei Bestimmung der spezifischen Leberclearance fanden wir nach Perfusion mit einem halbsynthetischen Medium und nach Perfusion mit verdünntem Rattenserum praktisch gleiche Werte für den Insulinabbau mit $0.18 \mathrm{ml} / \mathrm{min} / \mathrm{g} \mathrm{bzw} .0 .16 \mathrm{ml} / \mathrm{min} / \mathrm{g}$.

L-Leucin wurde von COCHRANE und Mitarbeitern [7] als kausal wirksames Agens bei einem Teil der idiopathischen Spontanhypoglykaemien der Kinder erkannt. Bei den späteren Untersuchungen konnte durch Leucin Hypoglykaemien bei Patienten mit $\beta$ Inselzelladenomen und, vor kurzem, auch bei Normalpersonen eine leichte, aber signifikante Blutzuckersenkung ausgelöst werden $[10,14]$. Die Möglichkeit einer physiologischen Blutzuckerregulation durch Leucin wurde diskutiert. Außer für L-Leucin konnte ein blutzuckersenkender Effekt auch für andere Aminosäuren nachgewiesen werden, so für Isoleucin, Alphaketoisocapronsäure, Valin u.a. Isovaleriansäure zeigte keine blutzuckersenkende Wirkung $[29,17$, $14,9]$.

Versuche, den Mechanismus der Leucinhypoglykaemie aufzuklären, haben bis jetzt zu keinem eindeutigen Ergebnis geführt. Bestimmungen des Insulins mit biologischen und immunologischen Methoden ergaben beim überwiegenden Teil der Patienten einen erhöhten Plasmaspiegel. Die größte Wahrscheinlichkeit kam der Erklärung zu, nach der der Anstieg des Seruminsulins durch eine Stimulation der Insulinfreisetzung aus dem Pankreas zustande kommen sollte. Dieser an sich naheliegende Schluß ließ sich jedoch in vitro durch Untersuchungen am isolierten Rattenpankreas nicht durch die erwarteten Ergebnisse belegen [26, 11]. Inkubation mit Leucin führte im Gegensatz zu Glucose zu keiner Erhöhung der Insulinabgabe durch das Rattenpankreas.

Die Möglichkeit, daß der Leucineffekt nicht an die Aminosäure selbst, sondern an eines der Abbauprodukte gebunden ist, erscheint ebenfalls unwahrscheinlich.

Aufgrund der Arbeiten von MADIsor et al. $[18,21]$ war insbesondere die Möglichkeit ins Auge zu fassen, daß der Leucineffekt auf dem Wege über die Endstufe des Leucinabbaus, das Acetoacetat, zustande kommt. Diese Arbeitsgruppe konnte nachweisen, daß die durch Ketokörper, speziell durch Acetoacetat, ausgelöste Hypoglykaemie durch eine verstärkte Insulinfreisetzung aus dem Pankreas verursacht wird. Von den Abbauprodukten des Leucins führt aber. nur die Alphaketoisocapronsäure zu einer regelmäßigen und in ihrem Ausmaß dem Leucineffekt vergleichbaren Hypoglykaemie [17, 14]. Diese Ketosäure kann aber durch Transaminierung leicht wieder in Leucin umgewandelt werden. Die nächste Abbaustufe, die Isovaleriansäure zeigte bei wiederholten Untersuchungen keine blutzuckersenkende Wirkung. Die folgenden Intermediärprodukte $\beta$-methyl- $\beta$-hydroxyglutarsäure und $\beta$-methylcrotonsäure ließen bei Verabfolgung an leucinempfindliche Kinder nur eine mäßige oder keine blutzuckersenkende Wirkung erkennen [15]. Da auch diese Intermediärprodukte zu Acetoacetat abgebaut werden, müßte eine dem Leucin äquimolare Menge auch zu einer vergleichbaren Hypoglykaemie führen.

Dieser Befund spricht ebenfalls gegen ein $\mathrm{Zu}$ standekommen des Leucineffektes auf dem Umweg über diese Intermediärprodukte. Darüber hinaus ist einzuwenden, daß dem Tyrosin, dessen Endprodukt ebenfalls das Acetoacetat ist, keine hypoglykaemische Wirkung zukommt [7] und zwischen dem Anstieg des 
Acetoacetats im Blut nach Leucingaben und dem hypoglykaemischen Effekt eine deutliche Diskrepanz besteht [9].

Neben einer direkten oder indirekten Erhöhung der Produktions- oder Sekretionsrate von Insulin unter dem Einfluß von Leucin wurde eine Potenzierung der peripheren Insulinwirkung erörtert, konnte aber experimentell nicht gesichert werden [16]. Wiederholt wurde ein hepatischer Effekt des Leucins in Erwägung gezogen $[8,26,14,28]$. Gegen eine Wirkung des Leucins auf den hepatischen Insulinabbau wendete man allerdings ein, daß Substanzen denen eine solche Wirkung in vivo zukommt, nie zu einem so deutlichen Blutzuckerabfall und Insulinanstieg im Plasma führen [14]. Dieser Einwand trifft jedoch für den geringen Leucineffekt im normalen Organismus nicht zu. Von MIRsKY wurde eine Beeinflussung der Insulinase durch Aminosäuren angenommen [23]. Cochrante fand nach Leucingaben eine Blutzuckersenkung in der Lebervene von Hunden [8].

Da die Leber bei einer einmaligen Durchströmung bis zu $50 \%$ des zugeführten Insulins inaktivieren kann [19] ließe sich der Leucineffekt im normalen Organismus durch eine Änderung dieser Größe hinsichtlich des Eintritts und Umfanges der Blutzuckersenkung und der Erhöhung des Insulinspiegels zwanglos erklären. Als Versuchstier war die Ratte geeignet, da sie ähnlich dem Menschen einer Beeinflussung des Blutzuckerspiegels durch Leucin unterliegt. Diese Beobachtung von Cochrane et al. [8] konnte von Pensuwan et al. [26] bestätigt werden, die nach intravenösen Leucingaben einen Blutzuckerabfall in Höhe von $20-27 \%$ des Ausgangswertes nachwiesen. Anscheinend widersprechende Befunde wurden von McArTHur et al. [20] mitgeteilt. Diese Ergebnisse stellen keinen wirklich stichhaltigen Einwand dar. Während des Versuchs wurde sowohl bei Kontrollen als auch nach Belastung der Ratten mit Leucin ein Blutzuckeranstieg nachgewiesen. Dieser Blutzuckeranstieg muß demnach wohl auf die Versuchsanordnung, beispielsweise das Abschneiden der Schwanzspitze zur Blutgewinnung bezogen werden.

Unsere Untersuchungen haben gezeigt, daß die Erhöhung des Seruminsulinspiegels als Ursache der Blutzuckersenkung nach Leucinzufuhr nicht durch Hemmung des Insulinabbaus in der Leber erfolgt. Die Leberelearance für Insulin in einem halbsynthetischen Medium betrug ohne Leucin $2.67 \mathrm{ml} / \mathrm{min}$, mit Leucin $2.18 \mathrm{ml} / \mathrm{min}$. Bei Berechnung der spezifischen Leberclearance ergab sich ein Wert von $0.18 \mathrm{ml} / \mathrm{min} / \mathrm{g}$ sowohl in den Kontrollperfusionen als auch nach Leucin. Demnach veränderte Leucin in unseren Versuchen die spezifische Leberclearance für Insulin nicht.

Die vorgelegten Befunde geben keinen Hinweis auf eine wesentliche Rolle der Leber beim Zustandekommen der Veränderungen des Plasmainsulinspiegels nach Leucingaben. Da Leucin die Insulinclearance der Leber nicht beeinflußt und da, wie bereits erwähnt, die Insulinsekretion des isolierten Rattenpankreas durch
Leucin nicht stimuliert wird, bleibt die Frage nach dem Wirkungsmechanismus dieser Aminosäure auf den Insulin- und Blutzuckerspiegel ungeklärt und bedarf einer weiteren Bearbeitung.

\section{Literatur}

[1] Begker, F.O., J. Clark and T.B. Schwarz: Lleucine sensitivity and glucose tolerance in normal subjects. J. clin. Endoer. 24, 554 (1964).

[2] BERSON, S.A., and R.S. YALOW: Radiochemical and radiobiological alterations of $\mathbf{I}^{\mathbf{1 3 1}}$ labeled proteins in solution. Ann. N.Y. Acad. Sci. 70, 56 (1957).

[3] Bolinger, R.E., and H.J. Grady: Plasma insulin${ }^{131} \mathrm{I}$ in diabetic patients. Ann. Int. Med. 48, 753 (1958).

[4] Bürgi, H., E.B. Ramseier, E.R. Froesch, P. Bally und A. LABHART: ,Freies" und, gebundenes": Insulin im Serum von Patienten mit B-InselzellAdenom. Helv. med. Acta 29, 527 (1962).

[5] - Kopexz K., K Schwarz und E.R. Froesch: Fate of rat insulin in rat-liver perfusion studied by adipose tissue assay. Lancet $\mathbf{1 9 6 3 / I I , ~} 314$.

[6] - W.A. Müller, R. E. Hưmbel, A. Labhart und E.R. FrotsCH : Non suppressible insulin-like activity of human serum. I Physico-chemico properties, extraction and partial purification. Biochim. biophys. Acta 1966 (erscheint in Kürze).

[7] Cochrane, W.A., W.W. Payne, M.J. Simpriss and L.J. WooLF: Familial hypoglycemia precipitated by amino acids. J. clin. Invest. 35, 411 (1956).

[8] - Studies in the relationship of amino acids to infantile hypoglycemia. A.M.A. Amer. J. Dis. Child. 99, 106/476 (1960).

[9] Fajans, S.S., J.C. Floyd, R.F. Knopf and J.W. CoNs: A comparison of leucine and acetoacatateinduced hypoglycemia in man. J. clin. Invest. 43, $2003(1964)$.

[10] Floyd, J.C., S.S. Fajans, R.F. KNopF and J.W. CoNa: Evidence that insulin release is the mechanism for experimetally induced leucine hypoglycemia in man. J. elin. Invest. 42, 1714 (1963).

[11] Frerichs, H., und W. Crevtzareidt: Die LeucinHypoglykämie. Dtsch. med. Wschr. 90, 960 (1965).

[12] Froesch, E.R., H. Bürgr, E.B. Ramseidr, P. Bally and A. LabHart: Antibody-suppressible and nonsuppressible insulin-like activities in human serum and their physiologic significance. J. clin. In. vest. 42, 1816 (1963).

[13] - - W.A. MüLler und A. Labhart: Mit Antiinsulinserum hemmbare und nicht hemmbare Insulinaktivität im menschlichen Serum. Schweiz. med. Wschr. 94, 309 (1964).

[14] Grumbach, M.M., and S.L. Kaplan: Amino acid and alpha-keto acid-induced hyperinsulinism in the leucine sensitive type of infantile and childhood hypoglycemia. J. Pediat. 57, 346 (1960).

[15] Hartmann, A. F', H.J. Wohltmann, J. Holowach and B.M. CALDwell: Studies in hypoglycemia. J. Pediat. 56, 211 (1960).

[16] Johnson, P., B. Herring and J.B. Field : Effects of L-leucine and its metabolites on glucose uptake by rat diaphragm. Metabolism 10, 415 (1961).

[17] Mabry, C.C., A.M. DI George and V.H. AUERBaCh: Leucine-induced hypoglycernia. J. Pediat. 57, 539 (1960).

[18] Madison, L.L., D. Mebane, R.H. Unger and A. LoofrNer: The hypoglycemic action of ketones. II. Evidence for a stimulatory feedback of ketones on the pancreatic beta cells. J. clin. Invest. 43, 408 (1964).

[19] - B. Combes, R.H. UNGER and N. Kaptax: The relationship between the mechanism of action of the 
sulfanylureas and the secretion of insulin into the portal circulation. Ann. N.Y. Acad. Sci. 74, 548 (1958).

[20] MoArthur, L.G., W.R. Krrthey and S.O. WAife: Effects of large doses of L-leucine in animals and man. Amer. J. clin. Nutr. 13, 285 (1963).

[21] Mebant, D., and L.L. Madison: Hypoglycemic action of ketones. I Effects of ketones on hepatic glucose output and peripheral glucose utilization. J. Lab. clin. Med. 63, 177 (1964).

[22] Mirter, L.L., C.G. Bux, M.L. Watson and W.F. BALE: The dominant role of the liver in plasma protein synthesis. A direct study of the isolated perfused rat liver with the aid of lysine-E-C ${ }^{14}$. J. exp. Med. 94, 431 (1951).

[23] Mirsky, J.A., G. Perisutit and D. Diengott: The hypoglycemic and insulinase inhibitory action of $\mathrm{L}$ tryptophan. Endocrinology 59, 369 (1956).

[24] Mortimore, G.E., and F. Tietze: Studies on the mechanism of capture and degradation of insulin-I ${ }^{131}$ by the cyclically perfused rat liver. Ann. N.Y. Acad. Sci. 82, 329 (1959).

[25] - - and D. Stertren: Metabolism of insulin-I'131. Diabetes 8, 307 (1958).

[26] Pensuman, S., F. Zacharewics, R. Yerry and T.F. Frawley: Studies on the mechanism of leucine-induced hypoglycemia. Metabolism 12, 773 (1963).

[27] Reaven, G., and C. Lucas: The use of insulin in the production of L-leucine-induced hypoglycemia in normal dogs. J. clin. Invest. 41, 654 (1962).

[28] C. WinkeLman and C. Lucas: Hypoglycemic effect of L-leucine during periods of endogenous hyperinsulinism. Diabetes 12, 205 (1963).
[29] Rosenthat, I. M., R. Metz and C. Pirani: Congenital leucine-sensitive hypoglycemia. A.M.A. Amer. J. Dis. Child, 107, 343 (1964).

[30] Samaax, N.A.: W.J. Dempster, R. Fraser, N.W. Please and D. Stillmax: Further immunological studies on the form of circulating insulin. J. Endocr. 24, 263 (1962).

[31] - - - and D. Stullmax: Immunological studies on two forms of circulating insulin. Proc. Biochem. Soc. $82,29(1962)$.

[32] - D. Strlliman and R. Fraser: Abnormalities of serum insulin-like activity in liver disease. Lancet 1962/II, 1287.

[33] - W.J. Dempster, R. Fraser and D. Stillman: Changes in levels of "atypical" circulating insulin after infusing "typical" insulin through the liver. J. Endocr. 26, 1 (1963).

[34] Schimassex, H.: Metabolite des Kohlenhydratstoffwechsels der isoliert perfundierten Rattenleber. Biochem. Z. 336, 460 (1963).

[35] Siess, E., A. Teinzer, E. STRUCK und O. WiELAND: Bildung eines insulinartigen Wirkstoffes durch die isolierte Rattenleber. Diabetologia 1, 21 (1965).

[36] Sonksen, P.H., J. Eutis, C. Lowy, A. Rutherford and J.D.N. NABARRo: A correlation between "sup. pressible" and "nonsuppressible" insulin-like activity and plasma insulin measured by radio immunoassay. Erste Tagung der Europäischen Gesellschaft f. Diabetologie in Montecatini 1965.

Priv.-Doz. Dr. K. Kopetz

II. Med. Klinik der Universität

8 München 15

Ziemssenstr. 1 